\title{
Acceleration errors in MBE-4 and in a driver.
}

\section{A.Warwick and E.P.Lee}

Two different acceleration schedules have been implemented in $\mathrm{MBE}-4$ and the resulting longitudinal emittance measured in each case. The question arises, whether this measured longitudinal emittance is small enough so that when scaled up to a driver, using induction pulsers of the same quality, the stringent constraint on $\Delta p / p$ at the final focus can be met.

In this note we first derive an expression for $\Delta p / p$ in terms of random voltage errors at the accelerating gaps. The uncorrelated acceleration errors acting, at each gap, on a particular slice of the bunch length, contribute to the final momentum spread like a random walk with a step size decreasing down the linac. The contribution from the beginning of the linac is largest because of subsequent acceleration and subsequent bunch shortening (see equation ).

Using known values for the voltage errors and kinetic energy in $M B E-4$ the derived expression for cumulative $\Delta p / p$ gives a value consistent with the measured value of the longitudinal emittance. We then extrapolate to a driver with the same accelerating pulsers and acceleration errors.

Since momentum is related to the accumulated voltage of the beam as

$$
p^{2}=2 m q e V
$$

the momentum error produced at the $n^{\prime}$ th gap is

$$
\frac{\text { gem } \Delta V_{n}}{p_{n}}
$$

where $P_{n}$ is the beam momentum at this gap and $\Delta V_{n}$ is the local voltage error. Simple shifts in the mean bunch energy and smooth changes in the velocity tilt of the bunch are not to be included in this voltage error. The ion charge is $q \cdot e$ and $m$ is the mass. By the end of the linac this error will be amplified due to bunch shortening by a factor 


$$
\ln / l_{f}
$$

where $l_{n, f} f$ is the bunch length at gaps $n, f$. Here we are neglecting collective effects due to space charge. The contribution at the end of the linac (gap f) from gap $n$ is then given by

$$
(\Delta p)^{2}=\left(\frac{l_{n}}{l_{f}}\right)^{2}\left(\frac{q e m}{p_{n}}\right)^{2}\left(\Delta V_{n}\right)^{2}
$$

Normalizing by the final momentum

$$
\begin{aligned}
& \left(P_{f}{ }^{2}=2 m q e V_{f}\right) \\
& \frac{(\Delta p)^{2}}{P_{f}{ }^{2}}=\frac{1}{4}\left(\frac{l_{n}}{l_{f}}\right)^{2} \frac{\left(\Delta V_{n}\right)^{2}}{V_{f} V_{n}}
\end{aligned}
$$

where $V_{n}, f$ is the voltage accumulated by the beam from the beginning of the linac to gaps $n, f$. Parameterizing the bunch length through the linac by

$$
l_{n} \sim V_{n}^{-1 / 4}
$$

which fits crudely to the usual driver design, using $m=200 a m u$ and $q=3$, in which the bunch length $\ell$ decreases from $60 \mathrm{~m}$ to $10 \mathrm{~m}$ as $V$ increases by a factor of $10^{3}$. This scaling law is somewhat pessimistic since a driver would use more vigorous compression at low velocity to reduce core costs.

Then

$$
\frac{(\Delta p)^{2}}{P_{f}^{2}}=\frac{1}{4} \frac{\left(\Delta V_{n}\right)^{2}}{V_{f}^{1 / 2} V_{n}^{3 / 2}}
$$

Assuming the intended voltage increments $(\delta V)$ are the same in all accelerating gaps, with the same random, uncorrelated error $\left(\eta_{n} \cdot \delta V\right)$ then the mean square momentum error at the end of the linac is 


$$
\begin{aligned}
\frac{\overline{(\Delta p f)^{2}}}{P f^{2}} & =\frac{1}{4} \sum_{n=1}^{N} \frac{\overline{\eta_{n}^{2}} \delta V^{2}}{V_{f}^{1 / 2} V_{n}^{3 / 2}} \\
& \simeq \frac{\overline{\eta^{2}} \delta V}{4} \int_{V_{i}}^{V_{f}} \frac{d V}{V_{f}^{1 / 2} V^{3 / 2}}
\end{aligned}
$$

There are $N$ accelerating gaps and

$$
\delta V=\left(V_{f}-V_{i}\right) / N
$$

Then

$$
\frac{\overline{\left(\Delta p_{f}\right)^{2}}}{p_{f}{ }^{2}}=\frac{\overline{\eta^{2}}}{2} \frac{\left(V_{f}-V_{i}\right)}{N} \frac{1}{V_{f}^{1 / 2}}\left(\frac{1}{V_{i}^{1 / 2}}-\frac{1}{V_{f}^{1 / 2}}\right)
$$

Since $V_{i} \ll V_{f}$ we have

$$
\overline{\frac{\left(\Delta p_{f}\right)^{2}}{P_{f}^{2}}} \simeq \frac{\overline{\eta^{2}}}{2 N} \sqrt{\frac{V_{f}}{V_{i}}}
$$

Figures 1 and 2 show the measured longitudinal remittance at the end of MBE-4 for the two acceleration schedules we have implemented. The first schedule accelerates and amplifies the current vigorously from $10 \mathrm{~mA}$ to $90 \mathrm{~mA}$ per beam. The second schedule is gentler, giving a final current of $35 \mathrm{~mA}$ per beam. In both cases systematic acceleration errors give rise to kinks in the phase space distribution, most noticeably at the head of the beam in the vigorous acceleration schedule. The widths of the distributions arise from shot-to-shot variations. We find the area of an ellipse enclosing the measured phase space distributions to be determined by the systematic errors in both cases and to be:

3 


$$
\pi \epsilon_{\text {longitudinal }}=4 \pi 10^{-3} \mathrm{eV} \mathrm{s}
$$

for the vigorous schedule

where we have ignored the large systematic error at the head of the bunch which could presumably have been corrected if additional pulsers had been available. And:

$$
\pi \epsilon_{\text {longitudinal }}=3 \pi 10^{-3} \mathrm{eVs} \quad \begin{aligned}
& \text { for the gentle } \\
& \text { schedule }
\end{aligned}
$$

What about the formula derived above? In MBE-4 (gentle schedule) we have

$$
V_{i}=200 \mathrm{kV} \quad V_{f}=620 \mathrm{kV} \quad N \simeq(620-200) / 20=21
$$

like this

The probability distribution of error amplitude looks something

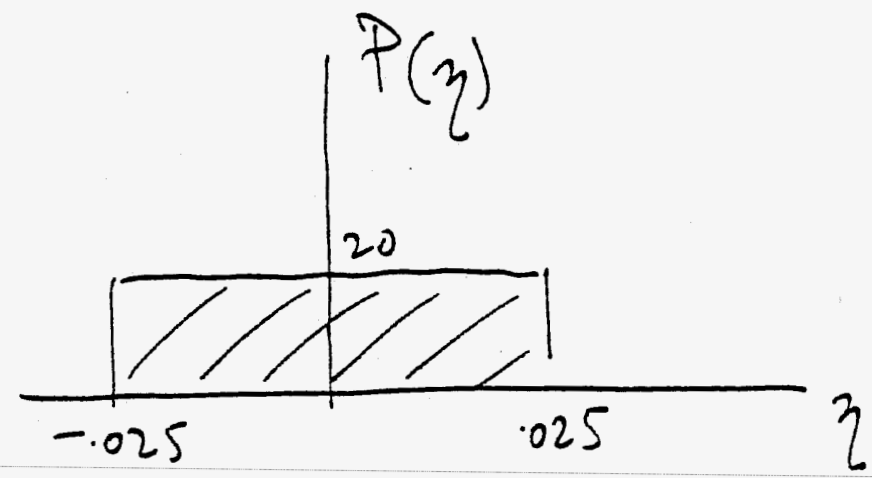

which will lead to current fluctuations of about $5 \%$, as observed. (Figure 3 shows measurements of the current waveform as the bunch passes diagnostic gaps throughout the linac under the gentle schedule). This distribution of errors gives

$$
\overline{\eta^{2}}=2.1 \times 10^{-4}
$$

giving

$$
\frac{\left(\Delta p_{f}\right)_{\text {r.m.s. }}}{P_{f}}=3 \times 10^{-3}
$$

4 
so at $620 \mathrm{keV}$

$$
(\Delta T)_{\text {r.m.s. }}=3.7 \mathrm{keV}
$$

and using $1.0 \times 10^{-6}$ as the overall duration of the bunch at the end of MBE-4 and twice the r.m.s. kinetic energy error (above) as the half width of the phase space distribution

$$
\pi E_{\text {lougitudinal }} \simeq 3.7 \pi 10^{-3} \mathrm{eV} \mathrm{s}
$$

which is close to the measured values.

In a driver with the same $20 \mathrm{kV}$ pulsers and the same acceleration errors as in MBE-4

$$
\begin{gathered}
V_{i}=3 \times 10^{6} \quad V_{f}=3 \times 10^{9} \quad N \simeq \frac{3 \times 10^{9}}{2 \times 10^{4}}=1.5 \times 10^{5} \\
\overline{z^{2}}=2.1 \times 10^{-4}
\end{gathered}
$$

giving a final momentum spread at the end of the linac, before drift compression of

$$
\frac{\left(\Delta p_{f}\right)_{\text {r.m.s. }}}{p_{f}}=1.5 \times 10^{-4}
$$

This is of the order of the allowed value of $10^{-4}$, set by the requirements of final focus following a further ten-fold bunch length compression after the linac.

It should be noted that in this scenario there is no phase space available for longitudinal emittance growth through the interaction of high current beams with the structure impedance. 
Pulsers of the quality installed on MBE-4 may be acceptable for the bulk of the acceleration in a driver, but more sophisticated correction pulsers would then be required at intervals down the linac. These must offer greater precision and would be used in conjunction with very careful monitoring of every pulser to correct velocity errors in the bunch before they oscillate into current fluctuations. These correction pulsers would thus remove systematic acceleration errors such as those seen in figures 1 and 2.

One would prefer to build a driver with fewer pulsers of larger amplitude than the $20 \mathrm{kV}$ typical of MBE-4. The formula for the r.m.s. momentum spread shows that the mean square fractional voltage error $\overline{\eta^{2}}$ must be reduced in proportion to the decreasing number of pulsers. 


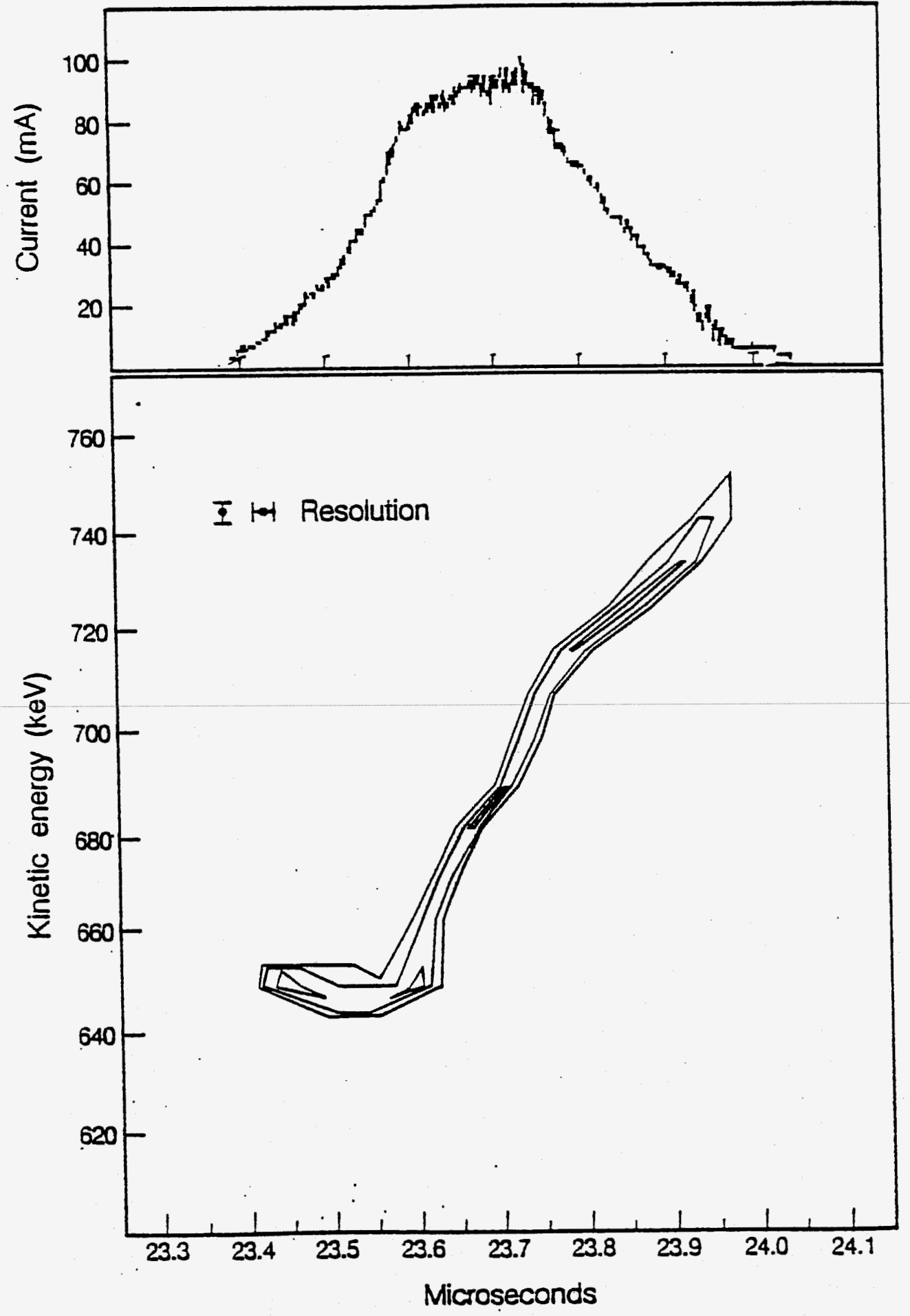




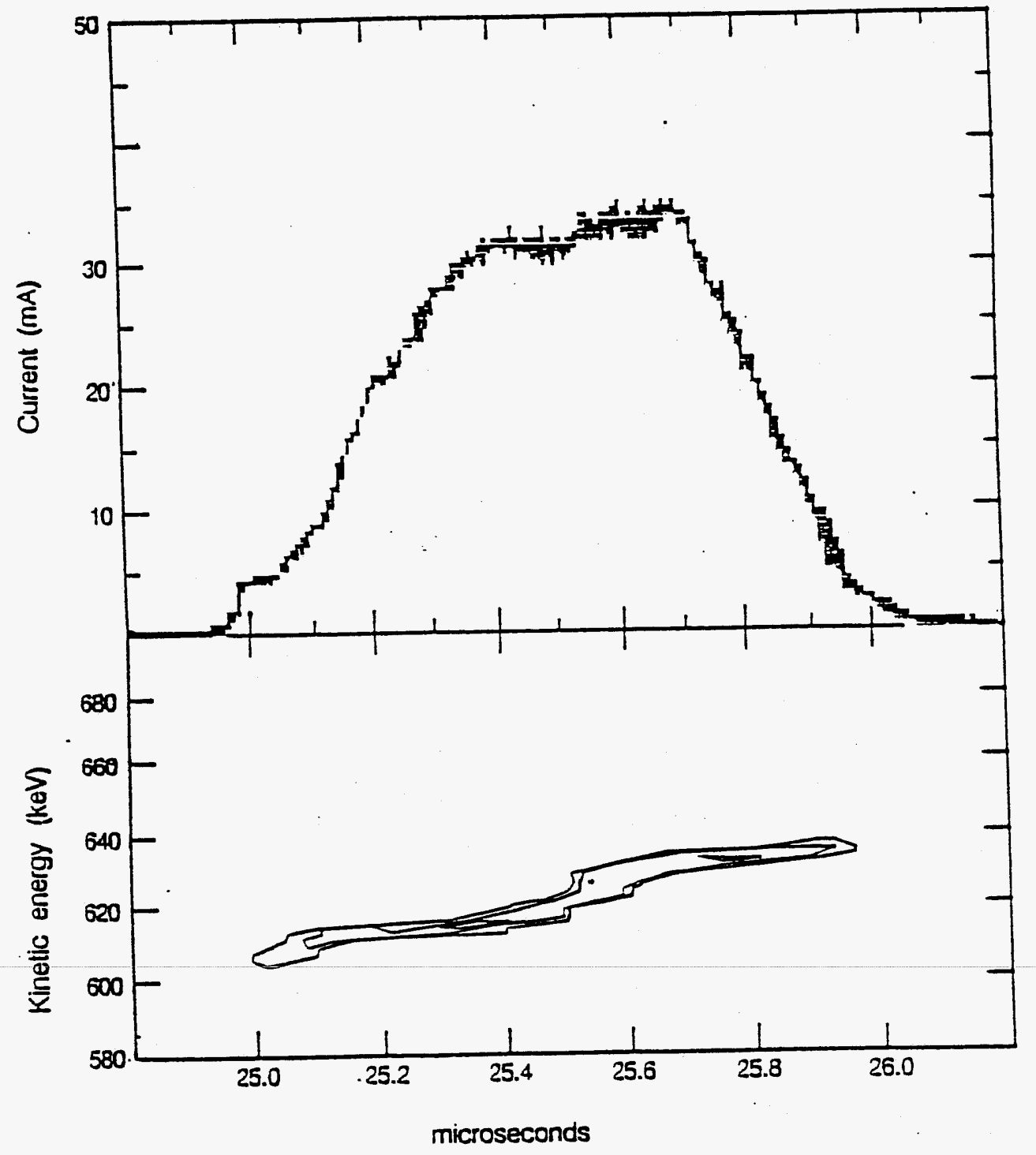

XEL 889.9728 


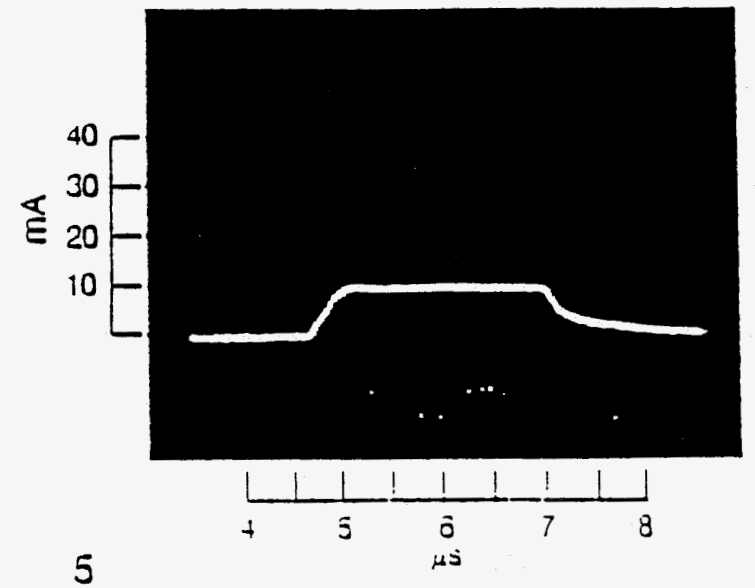

10
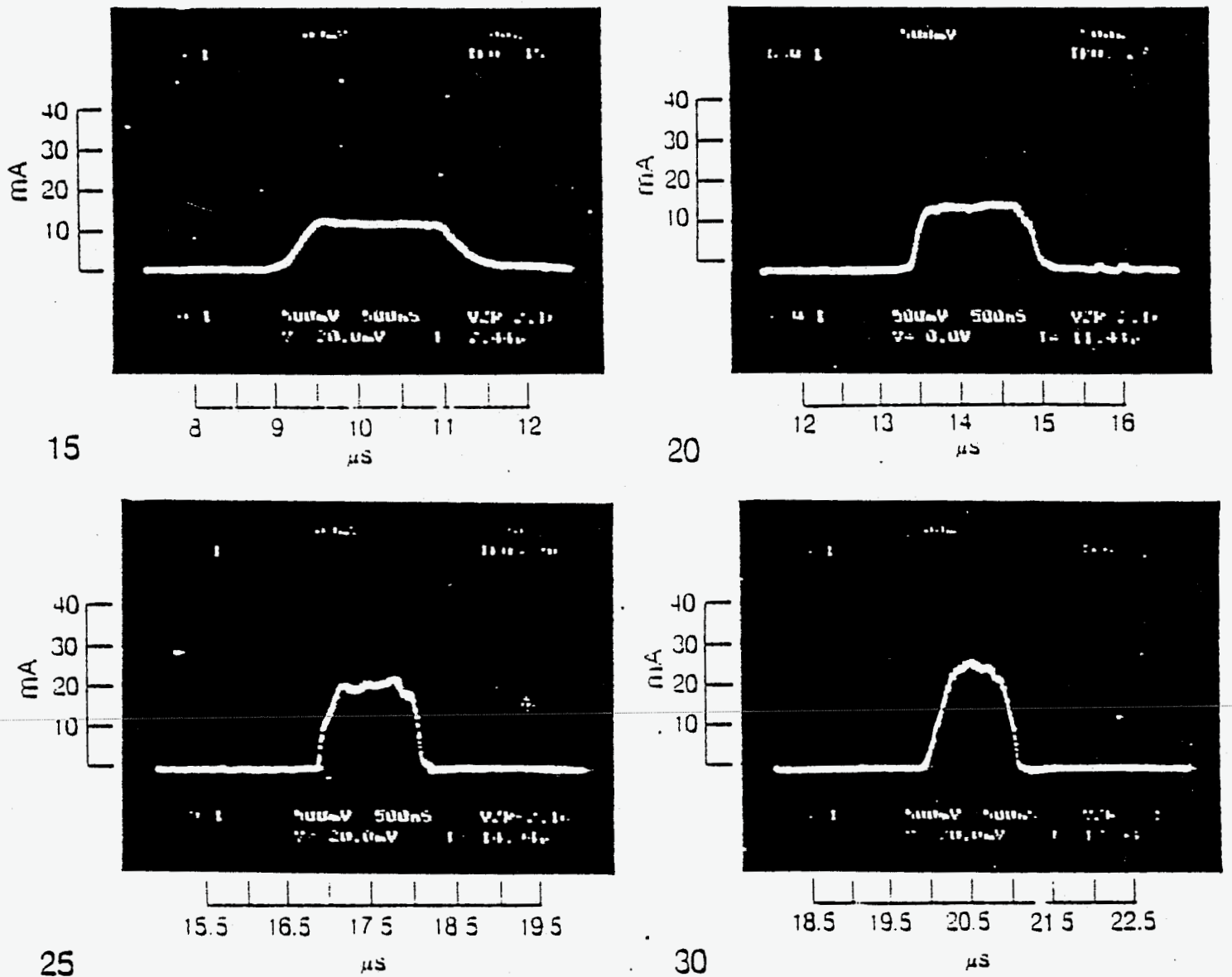

25
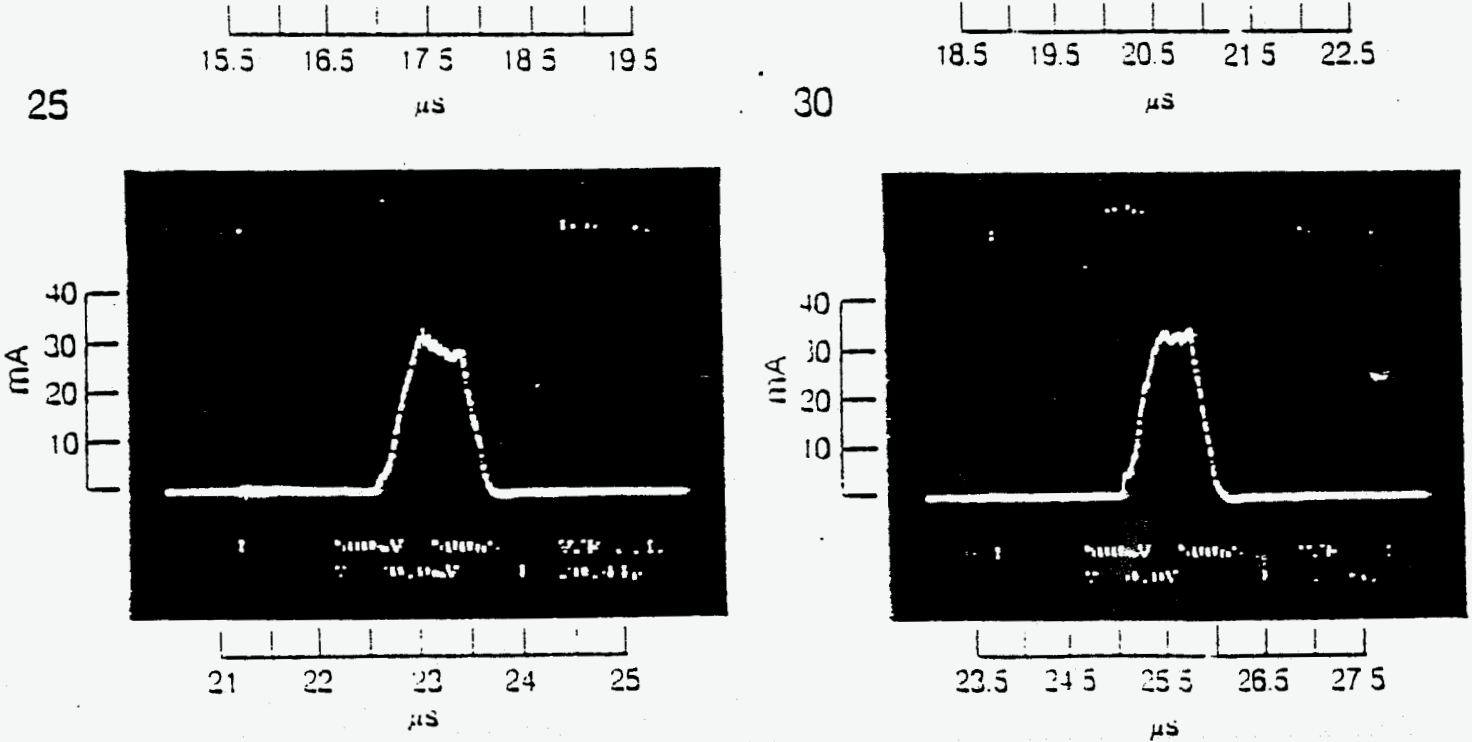

$\times 84.889 .9730$ 